Revue scientifique sur la conception et l'aménagement de l'espace

\title{
Retourner en forêt quinze ans après sa thèse : quelle place nouvelle pour le paysage dans la gestion forestière depuis le début des années 2000 ?
}

Returning to the Forest Fifteen Years after Completing a Thesis: The New Role of the Landscape in Forest Management since the Early 2000s

\section{Véronique Fourault-Cauët}

\section{(2) OpenEdition}

\section{Journals}

Édition électronique

URL : http://journals.openedition.org/paysage/8777

DOI : 10.4000/paysage. 8777

ISSN : 1969-6124

Éditeur :

École nationale supérieure du paysage de Versailles-Marseille, Institut national des sciences appliquées Centre Val de Loire - École de la nature et du paysage, École nationale supérieure d'architecture et de paysage de Bordeaux, École nationale supérieure d'architecture et de paysage de Lille, Agrocampus Angers

\section{Référence électronique}

Véronique Fourault-Cauët, « Retourner en forêt quinze ans après sa thèse : quelle place nouvelle pour le paysage dans la gestion forestière depuis le début des années 2000 ? », Projets de paysage [En ligne], 22 | 2020, mis en ligne le 21 juillet 2020, consulté le 24 juillet 2020. URL : http:// journals.openedition.org/paysage/8777 ; DOI : https://doi.org/10.4000/paysage.8777

Ce document a été généré automatiquement le 24 juillet 2020.

Projets de paysage 


\section{Retourner en forêt quinze ans après sa thèse : quelle place nouvelle pour le paysage dans la gestion forestière depuis le début des années 2000 ?}

Returning to the Forest Fifteen Years after Completing a Thesis: The New Role of the Landscape in Forest Management since the Early 2000s

Véronique Fourault-Cauët

1 Interroger la place du paysage dans les démarches et représentations des acteurs forestiers de plusieurs régions méditerranéennes : tel était l'objectif du travail doctoral achevé il y a 13 ans (Fourault-Cauët, 2007b). Alors que la faiblesse relative des productions sylvicoles dans ces régions laissait envisager la possibilité d'une émergence plus rapide des approches paysagères au sein des forêts méditerranéennes, leur conférant un rôle de laboratoire de la multifonctionnalité, le constat dressé avait été celui d'une relative frilosité des différents acteurs des zones étudiées (France, Espagne, Italie), à l'exception de la démarche menée par quelques individualités. Nous en avions alors déduit, tant pour des raisons structurelles que conjoncturelles, une relative difficulté à considérer le paysage comme un outil d'aménagement des territoires au sens plein du terme.

2 Notre proposition entend ici effectuer un "retour sur terrain » à l'image de ceux effectués par d'autres géographes (Rougé, 2011) afin de prendre la mesure des évolutions effectuées depuis la fin du travail doctoral. Si l'existence d'une « demande sociale » de paysage est connue de longue date (Luginbühl, 2001), la formalisation d'une demande sociale explicite autour des espaces forestiers, a fortiori méditerranéens, apparaissait limitée en 2007. Qu'en est-il désormais?

3 En prolongeant certaines des démarches qui avaient été alors menées, à savoir le dépouillement des revues forestières (Fourault-Cauët, 2007a), l'analyse des documents institutionnels (orientations régionales forestières, chartes forestières de territoire, 
etc.) susceptibles de se saisir de la question paysagère, et l'examen de l'offre de formation forestière quant au paysage, notre propos espère saisir les évolutions de la place accordée au paysage dans la gestion forestière, tout particulièrement en zone méditerranéenne française, et restreinte ici à la seule région Paca.

4 Si la question du paysage semble avoir acquis un droit de cité parmi les publications forestières et faire l'objet de références quasi systématiques dans les plans de gestion et les chartes forestières, le statut accordé aux problématiques paysagères et le sens donné au paysage dans la gestion forestière (s'agit-il d'une préoccupation limitée à quelques espaces telles les Forêts d'Exception ou bien d'un élément central de toute gestion forestière contemporaine ?) demeurent des interrogations d'actualité. La très difficile articulation de projets paysagistes, souvent réduits à une approche cosmétique de limitation des impacts visuels négatifs des opérations forestières et/ou de valorisation des sites les plus exceptionnels d'un point de vue esthétique, à l'aménagement plus global des territoires a été établie, en contexte forestier ou non (Davodeau, 2005 et 2011; Fourault-Cauët, 2007b). L'analyse des discours et des perspectives d'aménagement proposées par les documents analysés permettra ici de réévaluer cette difficulté structurelle mais aussi la possibilité de la dépasser. Le paysage est-il en passe de devenir un outil possible de la coconstruction de territoires forestiers à la multifonctionnalité revendiquée?

\section{Revue forestière et documents d'aménagement : analyse croisée de deux points de vue sur le paysage forestier}

5 Afin d'évaluer l'évolution de la part accordée au paysage dans les cercles forestiers depuis 2005, deux méthodologies ont été employées, en continuation des travaux antérieurement menés. Dans les deux cas, il s'agit d'évaluer la part du paysage dans les discours des acteurs forestiers, mais en choisissant des corpus aux différences notables. L'étude des articles publiés dans la Revue forestière française est menée pour mesurer la diffusion de la notion de paysage dans les travaux «théoriques » sur la forêt, relevant soit de travaux de recherche académique, soit de retours d'expériences sur des cas jugés pionniers, ou offrant des possibilités de diffusion. En parallèle, l'analyse des chartes forestières de territoire de la région Provence-Alpes-Côte d'Azur vise davantage à mesurer la place accordée au paysage dans des documents récents et à visée opérationnelle.

\section{Le dépouillement d'une revue forestière de référence}

6 Lors du travail doctoral, le choix d'un examen exhaustif des revues forestières de référence des pays étudiés avait été fait; dans le cas français, il avait été choisi d'examiner de manière systématique les articles de la Revue forestière française depuis sa création en 1949. Portée initialement par le principal organisme de formation forestière du pays, à savoir l'École nationale des eaux et forêts (de 1949 à 1964), puis par l'École nationale du génie rural, des eaux et des forêts (ENGREF, 1965-2006), avant d'être publiée par AgroParisTech à compter de 2007, la Revue forestière française constitue le principal canal de diffusion des réflexions des gestionnaires forestiers publics. Pour autant, nous y reviendrons, les dernières années se caractérisent par une 
ouverture non négligeable à des auteurs - et des approches - extérieurs au cadre des formations forestières et de l'office national des forêts (ONF).

7 Le dépouillement initial n'avait pu bénéficier d'un traitement par lexicométrie, compte tenu de l'absence totale de numérisation des documents et de l'usage de plusieurs langues dans le corpus utilisé à l'époque. Le choix avait été fait de procéder à une lecture cursive de l'ensemble des articles publiés, en analysant de manière détaillée les articles évoquant la question paysagère de manière explicite. Faute de pouvoir procéder à une recherche automatique, tous les articles publiés dans chacune des revues étudiées avaient été lus in extenso, afin d'y déceler l'éventuel usage du terme de « paysage » et des mots dérivés (paysager, paysagiste, paysager, etc.).

Bien que cette démarche soit moins précise qu'un logiciel de lexicométrie type IRaMuTeQ en matière d'analyse lexicale, il nous a semblé nécessaire de la réemployer pour étudier les évolutions enregistrables depuis 2005. Cela était indispensable pour pouvoir proposer une analyse reposant sur des bases identiques à celles employées durant le travail doctoral et permettant de ce fait une comparaison pertinente entre le premier corpus (1949-2005) étudié précédemment, et les publications recensées jusqu'à la fin de l'année 2018. Tous les articles évoquant la question du paysage ont été recensés indépendamment d'une mention des paysages méditerranéens. Articles théoriques sans localisation, mentions de forêts non méditerranéennes en France ou à l'étranger côtoient donc des textes relatifs aux espaces dont on étudiera en détail les politiques locales concernant les paysages forestiers.

Dans un second temps, la consignation sur un tableur Excel de la date de l'article, de ses auteurs et de leurs rattachements professionnels respectifs, des thématiques principales du texte parmi des catégories préétablies (gestion forestière, biodiversité, description régionale, etc.), des localisations mentionnées, des définitions du paysage éventuellement proposées de manière explicite, des valeurs attribuées à celui-là (connotation positive ou négative), etc. permet de dresser une tableau quantitatif de ces représentations.

10 Cette approche présente plusieurs limites: d'une part, en risquant d'omettre des articles où la question paysagère serait évoquée de manière implicite, d'autre part, en intégrant des textes où la réflexion paysagère apparaîtrait très marginale. Néanmoins, elle présente l'avantage de fixer un premier critère de délimitation du corpus assez clair, y compris dans un contexte plurilinguistique.

\section{Le dépouillement des chartes forestières de territoire d'une région méditerranéenne}

11 En parallèle au dépouillement de la Revue forestière française, nous avons choisi d'étudier la place dévolue au paysage dans les chartes forestières de territoire signées en région Paca depuis la loi d'orientation forestière de 2001 à l'origine de leur création. La charte forestière de territoire (CFT) est un instrument récent, explicitement pensé pour faciliter une approche territoriale cohérente des massifs forestiers: elle a donc une visée opérationnelle, même si l'échelle de référence apparaît plus petite que celle d'un plan simple de gestion. La CFT repose sur le principe d'une coopération de l'ensemble des acteurs de la forêt signataires, en prenant en compte la spécificité locale de ses usages (Riethmuller et al., 2003). Elle a été conçue pour structurer les territoires et les acteurs forestiers autour de l'activité sylvicole mais aussi d'autres formes de 
valorisation des boisements. En cherchant à promouvoir la multifonctionnalité forestière, les CFT peuvent donc apparaître comme un instrument privilégié de prise en charge des problématiques paysagères forestières. Notre propos est d'examiner cette hypothèse sur la période correspondant au corpus documentaire évoqué plus haut.

Depuis 2001, les initiatives de ce type se sont multipliées, pour atteindre aujourd'hui 15 chartes forestières de territoire ${ }^{1}$ rédigées et mises en œuvre en région Paca. Le choix de se concentrer sur la région Paca s'explique par la volonté de travailler sur des espaces où la faiblesse de la sylviculture et l'importance récréative des forêts peuvent constituer des facteurs favorables à l'émergence d'une prise en charge importante du paysage. La chronologie de ces documents, qui remontent au plus tôt au début des années 2000, apparait pertinente pour percevoir le développement des approches paysagères depuis cette date, les CFT ne pouvant par principe être la reconduction de documents antérieurs.

13 Nous avons donc examiné les textes de toutes ces chartes, qu'il s'agisse de la charte stricto sensu ou des fiches actions qui peuvent la compléter. Ces documents sont très inégaux, tant dans leur chronologie que dans l'importance des études menées : ainsi, leur volume varie de 12 à 239 pages, avec un degré de précision très fluctuant. De même, le nombre d'acteurs impliqués et le degré d'application du document varient d'une région à l'autre. Nous avons simplement exclu les chartes « en sommeil » d'après l'observatoire de la forêt méditerranéenne, de même que celles «en projet » et à ce jour non abouties. L'hétérogénéité forte du corpus doit être prise en considération, en interrogeant systématiquement son rôle éventuel dans les différences de représentations du paysage observées.

Comme pour la Revue forestière française, l'analyse des textes proprement dite repose sur le principe d'un examen des occurrences du terme de paysage et de ses dérivés dans les différentes chartes, puis d'une analyse détaillée des portions de texte consacrées au paysage: là aussi, ont été relevés les définitions explicites du terme lorsqu'elles existaient, les exemples localisés fournis, l'importance générale accordée au thème dans la charte (en relevant l'existence ou non de parties/fiches actions dédiées à la question paysagère ou pas, etc.).

Une partie des critiques évoquées plus haut quant à la moindre précision de ce type d'analyse face à une approche lexicométrique demeure ici. Pour autant, la combinaison d'un recensement quantitatif du terme de paysage, des lieux et des actions associées, et d'une lecture analytique des chartes permet de dégager plusieurs constats quant à la place accordée au paysage dans ces textes opérationnels. Ce faisant, nous reprenons pour partie des démarches croisées déjà éprouvées (Veschambre, 2007).

\section{Pourquoi croiser ces deux corpus}

Ces deux corpus de nature différente rendent compte de réflexions menées par plusieurs types d'acteurs du monde forestier, et répondant à des finalités variées. À cet égard, leur mise en regard peut s'avérer intéressante. Le corpus de la Revue forestière française permet de dresser un panorama de la diffusion des travaux scientifiques les plus récents sur la question paysagère parmi les gestionnaires forestiers, notamment publics, même si le lectorat de la revue s'est fortement diversifié au fil du temps. Les textes des chartes forestières sont $a$ contrario davantage élaborés par les acteurs de la forêt à échelle locale : gestionnaires forestiers, élus, propriétaires, etc. Ils renvoient 
donc potentiellement à d'autres approches de la question du paysage forestier. Les deux corpus peuvent donc présenter des convergences intéressantes, tout comme ils peuvent mettre en lumière des approches variées dans les conceptions, les pratiques paysagères et entre les acteurs. Ces points de rencontre et de disjonction sont tantôt internes à chaque corpus, tantôt perceptibles entre ces deux ensembles de documents.

17 L'hypothèse première à examiner lors de ce dépouillement est celle d'une part croissante accordée au paysage depuis 2005, accompagnant une mutation du sens qui est donné à ce terme au fil du temps par les différents auteurs. Il s'agit enfin d'interroger les types de représentations proposées dans ces deux cercles d'acteurs forestiers, tout en questionnant leur représentativité plus générale.

\section{De l'intérêt d'un retour sur objet : des modifications sensibles de la prise en compte paysagère par la Revue forestière française}

\section{Une troisième vague d'intérêt pour le paysage entre 2005 et 2018}

18 D'un strict point de vue numérique, l'examen exhaustif des numéros de la Revue forestière française parus depuis 2005 apparaît sans appel : en effet, on dénombre 64 articles évoquant la question paysagère entre 2005 et 2018, soit pratiquement la moitié de ce qui avait été enregistré entre 1949 et 2005 (127). Le graphique 1 présente en bleu les années étudiées durant la thèse, en vert celles examinées lors de ce «retour sur objet ». Il rend compte de cette évolution et permet de saisir trois vagues d'intérêt pour la question paysagère dans la revue : la première entre la fin des années 1960 et le début des années 1970, la deuxième durant les années 1990, et une troisième à la fin des années 2000. 
Figure 1. Nombre d'articles faisant référence au paysage dans la Revue forestière française depuis 1949

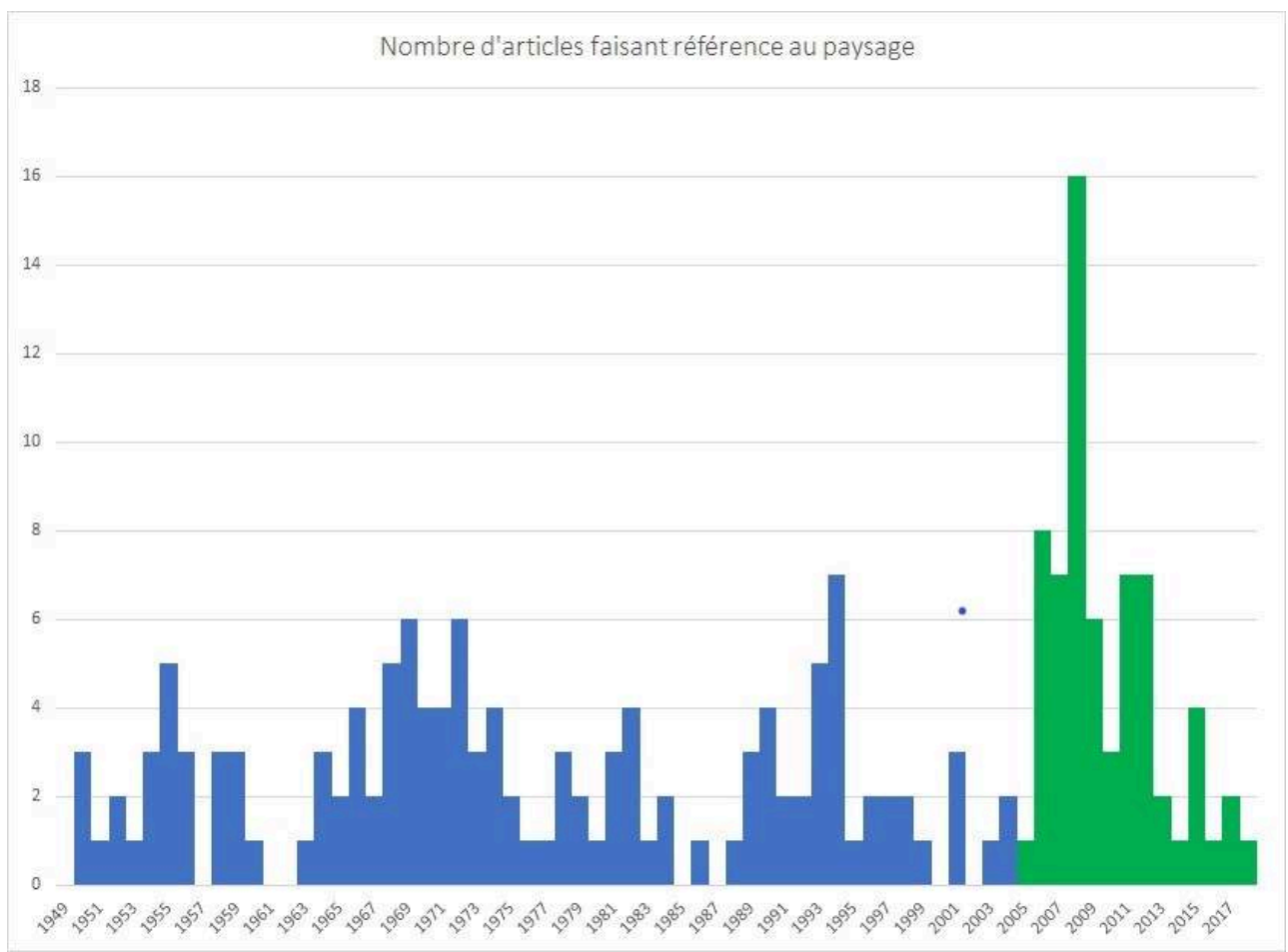

En bleu les années étudiées durant la thèse, en vert celles examinées lors du « retour sur objet ».

Source : Véronique Fourault-Cauët.

Ces trois phases correspondent à des formes d'intérêt variées pour le paysage : la première vague témoigne d'une prise de conscience des fonctions récréatives de la forêt de la part des gestionnaires de l'ONF durant les Trente Glorieuses; la deuxième peut s'expliquer par l'attention portée au paysage à la suite du vote de la loi Paysage de 1993 (Fourault-Cauët, 2007a). La troisième vague, objet plus spécifique de cet article, répondrait à d'autres logiques. L'explication proposée ici concernant cette troisième vague est en lien avec ce qui apparaît être une reconnaissance progressive de deux autres conceptions du paysage, tant d'un point de vue théorique que de gestion: celle de l'écologie du paysage et, de manière plus modeste, celle du paysage « cadre de vie » portée par la Convention européenne du paysage de Florence de 2001.

\section{Des paysages plus variés d'un point de vue théorique, mais de plus en plus désincarnés...}

L'analyse des différents textes parus entre 2005 et 2018 est révélatrice d'une diversification notable des approches paysagères évoquées dans les pages de la revue. On peut ainsi relever que 16 articles, soit près de $25 \%$ du corpus, abordent la question paysagère autour des problématiques de biodiversité. Plusieurs articles parmi ceux consacrés à la biodiversité privilégient une approche relevant de l'écologie du paysage, notamment dans le cadre de démarches de type Natura 2000. Des définitions du paysage en tant que notion centrale de cette approche sont d'ailleurs proposées, comme dans le texte de Roche et al. (2008) : 
«Le paysage est défini comme un système formé par une mosaïque de parcelles. L'unité élémentaire du paysage (UEP), ou élément, ou patch en anglais, est considérée comme une aire homogène au sens écologique, avec des limites précises au sens physique ou biologique.»

21 Le numéro thématique de 2008 coordonné par Gérard Balent et Marc Deconchat, tous deux membres de l'Inra et de l'UMR Dynafor, doit faire l'objet d'un examen spécifique : le numéro tout entier est en effet constitué des actes des $4{ }^{\text {es }}$ journées de l'International Association for Landscape Ecology-France (IALE-France, qui sera réintégrée à l'IALEEurope au cours des années 2000).Ce numéro explique en grande partie le pic d'articles observé en 2008, mais plusieurs articles postérieurs s'inscrivent dans des démarches scientifiques similaires et qu'il convient de rattacher à l'écologie du paysage, particulièrement dynamique durant cette décennie. L'introduction de ce numéro thématique (Balent et Deconchat, 2008) dresse un constat détaillé des inflexions récentes quant à la mise en œuvre d'une lecture paysagère des espaces forestiers :

«Aujourd'hui, le paysage visuel ne semble plus constituer un enjeu d'aménagement de l'espace aussi important qu'alors, sans doute du fait de l'émergence de questions et d'enjeux nouveaux qui semblent avoir plus de poids, comme la conservation de la biodiversité ou les services écologiques. Pourtant, les caractéristiques visuelles des paysages restent un outil de la recherche.»

Et de plaider pour une utilisation du paysage plus systémique, articulant notamment espaces agricoles et forestiers. De même, les auteurs concluent à la coexistence de recherches centrées tantôt sur la dimension visuelle du paysage, lue comme instrument de discussion entre les acteurs forestiers, tantôt sur la réalité fonctionnelle des écosystèmes.

De manière concomitante, les paysages examinés dans la Revue forestière française dans les toutes dernières années se singularisent par leur caractère paradoxalement de moins en moins strictement forestier. Plusieurs articles portent en effet non plus sur la forêt, mais sur les haies (par exemple Paletto et al., 2008, p. 437-450 ou encore Bécot et Gaborit, 2009, p. 535-538). S'y ajoutent des textes consacrés à l'arbre et aux forêts urbaines, déjà présents dans le corpus antérieur.

Cette diversification des types paysagers examinés s'accompagne d'un élargissement notable des échelles de la réflexion paysagère esquissée dans les pages de la revue. En effet, plusieurs articles insistent sur la nécessité d'une approche élargie, au-delà du seul contexte intra-forestier. Selon Rodolphe Schaepfer, professeur de l'Institut des sciences et techniques de l'environnement (École polytechnique fédérale de Lausanne) : « ... Gérer un système forestier dans le sens de l'approche paysagère signifie d'une part le placer dans un contexte spatial plus large, par exemple la région, et de tenir compte des influences des systèmes voisins comme les zones agricoles limitrophes ou les zones urbaines voisines ainsi que l'action sur les systèmes voisins du domaine forestier géré " (Schaepfer, 2007, p.515-523). L'introduction du numéro spécial de 2008, consacré à l'interface entre activités agricoles et forestières évoquée plus haut, souligne la nécessité d'échanges croissants entre acteurs ruraux sur la question paysagère; ce numéro en est lui-même le reflet puisqu'il rend compte d'un colloque ayant associé des acteurs multiples (écologues, gestionnaires, etc.) autour de cette question.

La géographie des espaces évoqués dans les textes est également révélatrice : si l'on se concentre sur les désignations de massifs forestiers, on peut relever une diminution nette des boisements emblématiques tels que Fontainebleau (cité 2 fois), ou, dans une moindre mesure, l'Aigoual (cité 4 fois), au bénéfice soit d'articles faisant référence à des 
terrains d'études qui ne relèvent pas des "hauts lieux" forestiers, soit, surtout, de textes sans localisation précise. C'est donc une géographie paysagère moins ancrée dans le territoire que précédemment, au bénéfice d'approches plus théoriques, dans lesquelles les espaces étudiés ne le sont pas au titre d'une valeur symbolique particulière. Cette nette inflexion en faveur des paysages "ordinaires" au sein de la Revue forestière française correspond à un mouvement plus général de prise en compte croissante des paysages comme composante quotidienne du cadre de vie. Formalisée par la Convention européenne de 2001, cette approche semble gagner en importance dans les pages de la revue, en élargissant la prise en compte paysagère à des paysages forestiers ou à composante forestière qui ne comptent ni parmi les panoramas les plus reconnus et fréquentés (ceux des grandes forêts domaniales périurbaines notamment), ni parmi les forêts longtemps considérées comme centrales dans le "cursus" de formation forestière (forêt de Tronçais, par exemple). Le corpus renvoie en partie aux dynamiques plus largement constatées d'un glissement du paysage conçu comme patrimoine, objet de rivalités dans ses manifestations les plus exceptionnelles, à une perception de celui-là comme bien commun à construire (Sgard, 2010). La référence aux régions des Comminges ou des Mauges dans plusieurs articles rend compte des travaux de plusieurs équipes de recherche, qui ont précisément choisi ces zones en raison du caractère peu emblématique de leurs paysages.

A contrario, le corpus apparaît singulier par l'absence notable d'articles à visée opérationnelle, répondant aux objectifs du paysagisme d'aménagement. Or, l'absence de textes présentant des «recettes " paysagères, jouant sur la forme de la lisière des boisements, les jeux de couleur des essences sélectionnées, ou encore les modalités de plantation (en banquettes ou non, etc.), est significative. C'est pourtant cette approche, largement diffusée durant les années 1980 par l'intermédiaire des guides techniques du Cemagref $(1976,1981)$, qui a été privilégiée lors de la constitution de la cellule paysage de l'ONF autour de Peter Breman, et a contribué à la formalisation de la question paysagère parmi les gestionnaires forestiers français. Pour autant, elle ne constitue pas un axe central des propositions en matière paysagère sur la période étudiée, sinon dans deux textes dressant une rétrospective des approches paysagères de la forêt (Deuffic, 2005 et 2006). L'auteur y pointe alors certaines des limites du paysagisme d'aménagement, dont on peut penser qu'elles expliquent pour partie sa faible présence dans les pages récentes de la Revue forestière française: faible proportion d'acteurs forestiers formés, défiance vis-à-vis des paysagistes, question du surcoût des opérations effectuées par rapport aux missions jugées «prioritaires» des forestiers. Plus fondamentalement, la difficulté à proposer une approche territorialisée de la forêt via des recommandations visuelles techniques nous semble pour partie explicative de cette faible théorisation du discours paysagiste appliqué à la forêt (Fourault-Cauët, 2007b).

En parallèle, de nouvelles approches du paysage - tout particulièrement l'écologie du paysage - gagnent en importance dans le corpus, notamment à la suite de travaux pionniers notamment entrepris dans les forêts étatsuniennes et canadiennes (Franklin et Forman, 1987 ; Forman, 1995 ; Boutin et Hébert, 2002) durant la décennie 1990. De ce point de vue, la Revue forestière française est représentative de la diffusion croissante des principes de l'écologie du paysage dans les cercles forestiers francophones à partir de la fin des années 1990 et du début des années 2000. De même, plusieurs articles mettent en avant l'intégration des problématiques de l'écologie du paysage dans les politiques de protection et de gestion de la biodiversité des années 2010 : c'est le cas du texte de Christian Barthod, Vincent Graffin et Michel Deshayes (2011, p. 307-322) ou de celui de 
François, et Lucile Rambaud (2011, p. 527-537). Leurs auteurs sont alors souvent en poste dans les ministères en charge de la mise en œuvre de ces politiques, y compris dans les territoires forestiers.

Ainsi donc, le dépouillement d'une décennie supplémentaire de la Revue forestière française se révèle instructif quant à l'évolution du rapport entre paysage, forêts et forestiers. Il met en évidence un regain d'intérêt pour la notion, mais autour d'approches plus clairement associées à l'écologie et à la biodiversité qu'auparavant. Les articles sur le paysage rassemblent des auteurs aux profils plus variés, et qui ne limitent pas leur approche à la stricte prise en compte des boisements : le territoire dans lequel s'inscrit la forêt est bien souvent pris en compte. Si le corpus étudié jusqu'à 2005 avait infirmé l'hypothèse d'un paysage comme instrument reconnu et opératoire d'aménagement de la forêt, le dépouillement des articles publiés entre 2005 et 2018 appelle à nuancer ce constat.

\section{... Mais en trompe-l'œil ? Les limites du travail sur une revue}

Pourtant, plusieurs éléments, tant d'un point de vue méthodologique que sur le fond de l'analyse, invitent à nuancer ces résultats.

Ainsi, l'inflation du nombre d'articles évoquant le paysage dans la Revue forestière française interroge: on passe en effet d'une moyenne de 2,26 articles par an sur la période 1949-2005 à 5,33 articles par an sur la période 2006-2018. Il convient d'examiner les facteurs possibles de cette augmentation, au-delà d'un intérêt croissant pour cette thématique de la part des acteurs forestiers.

31 Dans un premier temps, on peut envisager un potentiel biais dans la lecture des textes, qui déboucherait sur la prise en compte indue d'articles au regard de ce qui avait été sélectionné lors de la thèse : peut-on penser examiner un texte de la même manière à 15 ans d'intervalle? Néanmoins, le fait de poser comme condition sine qua non de l'intégration d'un article au corpus la présence du terme de "paysage " nous semble limiter les biais possibles, tout du moins pour ce qui relève de la comptabilisation des textes.

La publication de plusieurs numéros thématiques concourt en revanche largement à expliquer l'inflation numérique du nombre d'articles recensés. Ainsi, en est-il du numéro thématique de 2008 portant sur «Le paysage à l'interface des activités agricoles et forestières ", qui rassemble à lui seul 12 textes évoquant la question du paysage autour des problématiques forestières. C'est, dans une moindre mesure, également le cas des numéros relatifs à l'aménagement durable des forêts ou à la biodiversité.

Il faut également faire la part des choses entre les évolutions concernant la thématique paysagère et les mutations plus générales de la revue, mutations qui dépassent le cadre des seuls textes consacrés au paysage. Sur la période 2005-2018, les profils des auteurs se sont en effet diversifiés, avec une part désormais clairement minoritaire des gestionnaires forestiers issus de l'ONF, au profit des scientifiques et fonctionnaires en poste aux ministères de l'Agriculture, de l'Environnement ou de la Transition écologique suivant les périodes. Cette inflexion nous semble particulièrement révélatrice d'une ouverture tant de la revue que du monde forestier à des discours extérieurs à l'ONF. Le site Internet de présentation de la revue confirme cette ouverture nouvelle puisqu'il est expliqué que [des auteurs] « peuvent [...] être sollicités, 
notamment dans le cadre de numéros spéciaux ou à thèmes, qui constituent une œuvre collective, ou lorsqu'il s'avère opportun de faire s'exprimer des voix extérieures au monde forestier traditionnel ${ }^{2} »$. Cette diversification des intervenants au sein de la revue a contribué à la variété croissante des approches du paysage qui y sont développées.

L'analyse menée dans la Revue forestière française permet de conclure à une sollicitation croissante de la notion de paysage par les auteurs de cette publication spécialisée, en privilégiant de plus en plus une double approche de cette notion, davantage tournée vers l'écologie du paysage, mais aussi vers la prise en compte du paysage comme composante du projet territorial. Pour autant, peut-on observer une matérialisation de ces travaux théoriques dans les instruments concrets de gestion des espaces forestiers?

\section{Au-delà des apports théoriques, quel renouvellement des approches paysagères à échelle fine?}

Les différentes chartes forestières de territoire élaborées en Paca sont des textes dont les objectifs diffèrent de ceux de la Revue forestière française. Ces documents sont conçus avant tout comme devant favoriser l'élaboration d'un projet de territoire. Ce faisant, ils nous permettent d'appréhender dans une petite mesure la place consacrée au paysage dans ces projets, à l'instar de ce qui a pu être observé par ailleurs (Davasse et Henry, 2015).

\section{Les chartes forestières et le paysage : une attention croissante avec le temps?}

Figure 2. Chronologie des chartes forestières de territoire en région Paca

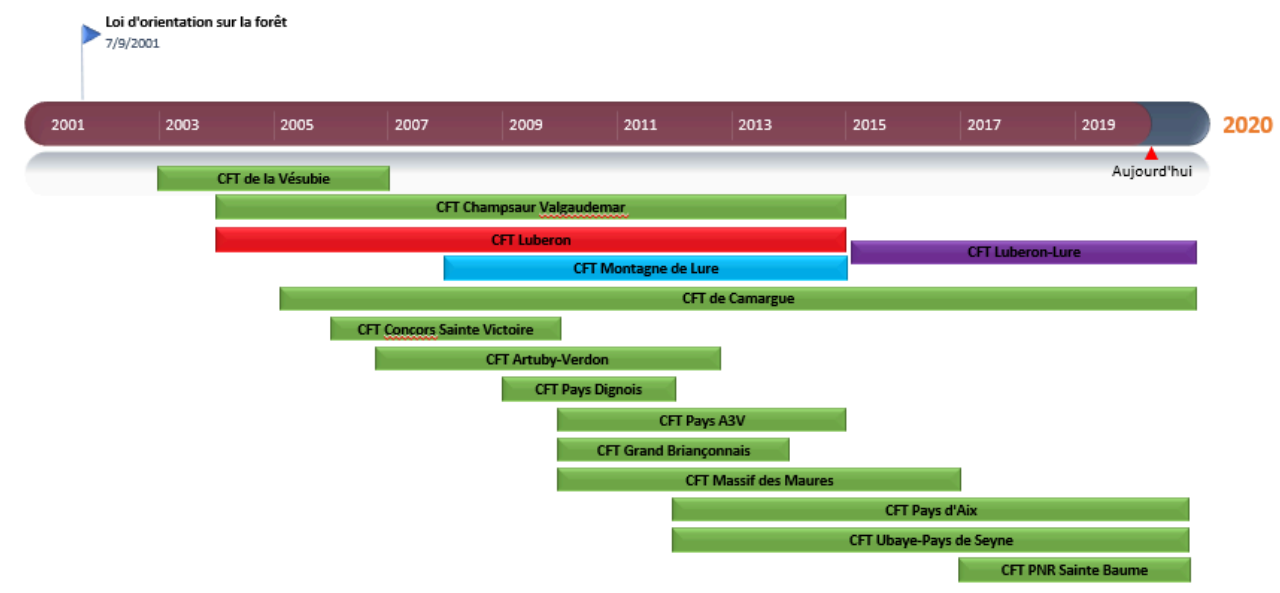

La charte de la réserve de biosphère Luberon Lure est la fusion de deux chartes antérieures (Montagne de Lure et Luberon).

Source : Véronique Fourault-Cauët.

La chronologie indiquée est indicative, car les chartes ont été bien souvent précédées de longues négociations, et leur effectivité peut varier au fil du temps. Néanmoins, plusieurs éléments ressortent de cet examen. Les quinze chartes étudiées présentent une chronologie très disparate, qui n'est pas sans incidence sur l'importance accordée 
au paysage : les chartes les plus anciennes - qui sont bien souvent les moins détaillées n'accordent le plus souvent que peu d'importance à la notion de paysage, même si des contre-exemples existent. La CFT de la Vésubie, mise en œuvre en 2002, n'y fait pas référence. Celle de la Camargue se concentre surtout sur la description paysagère des bosquets d'arbres, notamment sur les berges fluviales. La CFT du Pays des Maures ${ }^{3}$, signée en 2010 après 8 ans de consultation, ne fait à aucun moment référence au paysage forestier. Les deux priorités d'aménagement forestier et de gestion des boisements de ce dernier document sont explicitement la valorisation économique des massifs, et la défense contre les incendies très fréquents dans ces boisements littoraux touristiques.

En revanche, la plupart des chartes forestières de territoire récentes, qui concernent davantage les espaces périurbains et touristiques, sollicitent volontiers la notion de paysage. Deux chartes mentionnent des enquêtes de terrain autour des attentes paysagères des usagers de la forêt. C'est le cas de la CFT de l'Ubaye-Pays de Seyne, signée en 2012, qui présente les forêts comme "répond[ant] donc à une demande sociale marquée comme paysage quotidien ou de loisir ", et évoque la réalisation prochaine d'une charte paysagère dans le cadre de la labellisation "territoire d'art et d'histoire " du pays SUD [Serre-Ponçon Ubaye Durance]. La charte forestière expose que " cette "forêt paysage" demande aussi de repenser certaines actions sylvicoles afin d'en limiter les impacts négatifs (réflexion sur la localisation et le type des coupes, sur la création de nouvelles dessertes...) et de communiquer autour des activités sylvicoles afin qu'elles soient mieux perçues et acceptées par les visiteurs et les habitants ». C'est enfin et surtout le cas de la CFT Grand Estérel, signée en 2014. Cette charte renvoie à une enquête sur la valeur paysagère des lieux mais propose en outre la définition d'unités paysagères de plus grande échelle (les "micro-paysages »), et détaille des traitements spécifiques tels que le débroussaillement en peau de léopard ${ }^{4}$, à privilégier dans les espaces aux enjeux paysagers les plus forts. Il est ainsi exposé qu'il y a « un enjeu fort, aussi bien en termes de développement économique (tourisme) que de préservation du cadre de vie, à réduire ces points noirs paysagers et à préserver les micro-paysages. Sans "figer" les choses, l'essentiel est bien de conserver les "impressions", cultiver les "différences" et mettre en valeur cette diversité paysagère ». La notion de paysage est présentée dans le document comme centrale, en partie dans la perspective de l'élaboration d'une Opération grand site (OGS) ${ }^{5}$ dont le principe sera validé en 2018. La charte et les documents ultérieurs sur l'OGS privilégient dans ce cas une approche fondamentalement visuelle du paysage qui est privilégiée, renvoyant davantage aux principes du paysagisme d'aménagement en partie disparus du corpus de la Revue forestière française.

Pour autant, la chronologie de rédaction de ces documents n'explique pas tout; les profils des territoires concernés par les chartes expliquent pour beaucoup les différences enregistrées d'un texte à l'autre.

\section{Le paysage : une préoccupation des chartes forestières de territoires touristiques et résidentiels}

Plusieurs chartes forestières de territoire, qui ne sont pas forcément parmi les plus récentes, se distinguent par la très grande place accordée au paysage. C'est le cas de la CFT Concors Sainte-Victoire (2006), qui correspond à un paysage dont la valeur 
emblématique est reconnue de longue date tout comme celui du Luberon, évoqué dans la CFT Luberon Lure. La question paysagère est plus développée dans les chartes forestières de territoires à forte dimension résidentielle et touristique : ainsi, la CFT Champsaur Valgaudemar propose-t-elle de "promouvoir une gestion sylvicole paysagère des domaines skiables boisés (publics et privés)» en 2006 tandis que la CFT Grand Estérel, qui mobilise le plus le paysage, rappelle que « le tourisme est la première activité du territoire ». La CFT Pays d'Aix, qui couvre un espace marqué par une très forte fonction résidentielle, notamment du fait des extensions périurbaines massives autour d'Aix-en-Provence, mobilise également la notion de paysage de manière récurrente, en insistant tout particulièrement sur la dimension "quotidienne » des « micro-paysages".

Contrairement à ce qui avait pu être observé dans la Revue forestière française, les paysages évoqués dans les chartes forestières de territoire sont souvent très incarnés, précisément décrits et jugés. Certains paysages sont connotés positivement : c'est le cas des paysages "ouverts", très valorisés dans les chartes forestières des territoires de montagne. Ainsi, est-il expliqué dans la CFT Grand Briançonnais que «les enjeux, menacés par l'enfrichement des anciens espaces pastoraux, qu'ils soient paysagers ou patrimoniaux dans les vallées ou plus liés à la préservation des espèces et habitats, justifient des travaux de reconquête d'anciens espaces pastoraux. Il s'agit de travaux de défrichement préalables à la réinstallation d'éleveurs ». La CFT Ubaye Pays de Seyne indique de manière similaire : «Une autre problématique a été souvent évoquée tout au long de l'élaboration de cette charte. Il s'agit du pastoralisme et de l'élevage, une activité importante sur notre territoire pour l'économie locale avec encore de nombreux agriculteurs-éleveurs sédentaires qui contribuent pleinement au maintien d'un territoire ouvert, en améliorant ainsi la richesse paysagère. " En revanche, des chartes comme celles de Concors Sainte-Victoire, du Luberon ou du Pays d'Aix privilégient d'autres références paysagères, notamment le "patrimoine bâti » et le « patrimoine culturel ». Ce faisant, ces différentes chartes renvoient à la complexité des modèles paysagers méditerranéens qui reposent sur la coexistence de représentations des territoires comme étant tantôt sauvages, tantôt maîtrisés de longue date par les sociétés (Luginbühl, 1992). L'hétérogénéité des représentations paysagères valorisées est ici révélatrice, d'une part, de l'ambition commune des chartes de mettre en valeur "l'identité » (CFT Grand Estérel) des territoires par le paysage et, d'autre part, de la place très ambiguë de la forêt dans cette construction. De même, les représentations associées aux paysages de forêts résineuses sont particulièrement hétérogènes: les boisements en pins sont ainsi jugés producteurs de paysages " "non conformes" aux standards de la Haute-Provence ", tandis que les CFT du Champsaur ou du Grand Briançonnais proposent de protéger et de valoriser les paysages marqués par la présence de mélèzes : une partie de ces différences tient à l'évidence à des contextes géographiques très variés, depuis les paysages agricoles provençaux jusqu'aux zones d'altitude bien plus marquées, dont les composantes et la structure diffèrent matériellement.

41 Ce sont aussi les espaces et les boisements les plus emblématiques qui bénéficient de cette attention, dans une perspective touristique et résidentielle affichée. Ainsi, la charte du Parc naturel régional du Luberon développe-t-elle surtout le paysage forestier comme "cadre qui met en valeur de nombreux villages et monuments protégés ", et propose une action spécifique d'aménagement de sentiers dans la forêt 
des Cèdres de Bonnieux, haut lieu touristique du Luberon. L'évocation de paysages plus ordinaires est rare, essentiellement centrée sur la «fermeture » de ceux-là, présentée de manière très négative (CFT Lure, CFT Grand Briançonnais).

In fine, l'examen de ces différents documents, qui contractualisent l'engagement des acteurs locaux autour d'une action forestière territorialisée, invite à une lecture contrastée de l'usage du paysage comme élément d'action sur les territoires à fort enjeu forestier.

De manière générale, les CFT qui fixent davantage les grands objectifs du territoire de projet qu'elles ne proposent de solutions opérationnelles détaillées, accordent une place certaine et croissante à la notion de paysage. Pour autant, cette préoccupation demeure centrée sur une approche diagnostique de l'existant, de manière conforme à ce qui est observé hors du seul cadre forestier (Davasse et Henry, 2015). Par ailleurs, l'attention forte portée au paysage reste surtout l'apanage de territoires fortement marqués par l'activité touristique, et suivant une logique patrimoniale de valorisation des panoramas exceptionnels. Celle accordée aux espaces et paysages ordinaires demeure plus modeste, quoique croissante. Elle n'est jamais posée comme centrale, face à des enjeux comme ceux du risque incendie ou de la valorisation économique des bois.

Enfin, la définition donnée au terme de paysage dans ces différents textes apparait assez éloignée de celle proposée dans les textes les plus récents de la Revue forestière française : si l'on excepte les chartes forestières de territoires de montagne, bénéficiant par ailleurs souvent du statut d'espaces protégés (Parc national des Écrins, Parc naturel régional du Queyras, etc.) et étudiés par des écologues à ce titre, rares sont les textes qui développent une réflexion paysagère associée à la biodiversité et à l'écologie du paysage.

\section{Conclusion : rendez-vous dans dix ans?}

L'examen de ces deux corpus que sont la Revue forestière française et les chartes forestières de territoire de la région Paca depuis une quinzaine d'années permet de rendre compte d'un certain nombre d'évolutions quant à la prise en compte du paysage dans le monde forestier. La notion de paysage, quoique représentant toujours un « enjeu flou » (Deuffic, 2006) pour les services forestiers, n'a pas été abandonnée par les acteurs de la forêt dans leur ensemble. Le paysage est au contraire devenu un élément de réflexion fort pour penser l'articulation de la forêt avec ce qui lui est extérieur : acteurs non gestionnaires, qu'ils soient publics ou privés, territoires non forestiers mais en lisière des boisements. De même, le paysage est devenu un instrument manifeste d'intégration - parmi d'autres - des problématiques de biodiversité dans la sphère forestière, tout particulièrement par le biais de l'écologie du paysage. Reste que sur le terrain, ou du moins dans les documents à visée opérationnelle plus immédiate, la question paysagère demeure relativement secondaire, et cantonnée à des espaces où les regards sont particulièrement insistants : espaces touristiques emblématiques, espaces résidentiels pour lesquels la qualité du cadre de vie est une exigence forte. Les pistes de recherche sur l'articulation entre paysage et forêt détaillées dans la Revue forestière française ne semblent pas trouver d'écho très fort dans les projets de territoires forestiers constitués à la même période. Les résultats obtenus, s'ils confortent en partie ceux de la thèse de 2007 pour ce qui est de la place réduite accordée au paysage dans l'émergence de projets de territoires forestiers, montrent aussi que la critique du 
paysagisme d'aménagement n'a pas fait disparaître toute préoccupation paysagère des cercles forestiers. À cet égard, ce «retour sur objet» invite à poursuivre ces investigations sur le temps long, afin de pouvoir espérer saisir des évolutions lentes, qu'il s'agisse des recherches théoriques sur le paysage comme des représentations des paysages forestiers concrets. Si les structures paysagères évoluent parfois rapidement au gré des constructions ou de l'abandon agricole et pastoral, les rapports au territoire changent quant à eux plus lentement. Redéfinir la place des forêts et de leurs paysages dans les territoires ne se fait que sur le temps long, ce qui nous semble rendre pertinent(s) ce(s) retour(s) périodique(s )sur un objet de recherche antérieur.

\section{BIBLIOGRAPHIE}

Balent, G. et Deconchat, M., « Le paysage à l'interface des activités agricoles et forestières. Une introduction ", Revue forestière française, 2008, $\mathrm{n}^{\circ}$ 5, p ; 515-524.

Barthod, Christian ; Graffin, Vincent ; Deshayes, Michel, « Les trames verte et bleue. Un projet du Grenelle de l'environnement qui concerne les forestiers ", Revue forestière française, $n^{\circ} 3,2011$, p. 307-322.

Bécot, A. et Gabory, Y., « L'arbre rural créateur de paysage et de cadre de vie. Retour sur quinze ans d'expérience dans les Mauges (Maine-et-Loire) ", Revue forestière française, $n^{\circ}$ 5, 2009, p. 535-538.

Bland, F. et Rambaud, L., « Le réseau Natura 2000 : un défi pour la gestion multifonctionnelle des forêts ", Revue forestière française, $\mathrm{n}^{\circ}$ 5, 2011, p. 527-537.

Boutin, S. et Hébert, D., « Landscape ecology and Forest Management : Developing an effective partnership ", Ecological Applications, vol. 12, n 2, 2002, p. 390-397.

Cemagref, «Intégration des lignes électriques à haute et très haute tension dans le paysage forestier », Nogent-sur-Vernisson, note technique, $\mathrm{n}^{\circ}$ 52, 1986, $143 \mathrm{p}$.

Cemagref, « Reboisement et paysage », Nogent-sur-Vernisson, note technique $\mathrm{n}^{\circ} 45,1981,112 \mathrm{p}$.

Cemagref, « Forêt et récréation, prise en compte des facteurs "paysage" et "récréation"dans l'aménagement forestier ", Nogent-sur-Vernisson, note technique $\mathrm{n}^{\circ} 32,1976,47 \mathrm{p}$.

Davasse, B. et Henry, D., « Le paysage au cœur des projets de territoire », 2015, 12 p., : URL : https://halshs.archives-ouvertes.fr/halshs-01270407/document.

Davodeau, H., « La dimension spatiale de l'action paysagère ", Annales de géographie, $\mathrm{n}^{\circ} 679$, 2011/3, p. 246-265, URL : https://www.cairn.info/revue-annales-de-geographie-2011-3page-246.htm

Davodeau, H., « La sensibilité paysagère à l'épreuve de la gestion territoriale. Paysages et politiques publiques de l'aménagement en Pays de la Loire ", thèse de doctorat en géographie, université d'Angers, 2005, 303 p.

Deuffic, P., « Après les priorités du jour, le paysage... Enquête sur un enjeu flou pour les services forestiers ", Revue forestière française, $\mathrm{n}^{\circ} 3$, 2006, p. 229-244. 
Deuffic, P., « Les forestiers et la question du paysage. Des bois marmentaux à la forêt "paysagée" ", Revue forestière française, n³, 2005, p. 349-362.

Fourault-Cauët, V., « Le paysage, outil de territorialisation et d'aménagement incomplet pour les forêts méditerranéennes ?», Annales de Géographie, n 673, 2010/3, p. 268-292, URL : https:// www.cairn.info/revue-annales-de-geographie-2010-3-page-268.htm

Fourault-Cauët, V., « Le paysage dans trois revues forestières européennes : une notion consensuelle, des panoramas variés ?", Revue forestière française, $\mathrm{n}^{\circ}$ 4, 2007a, p. 379-392.

Fourault-Cauët, V., « Le paysage : notion théorique ou outil d'aménagement émergent pour les forêts méditerranéennes en Europe ? », thèse de doctorat de Géographie, université Paris 1 Panthéon-Sorbonne, 2007b, $490 \mathrm{p}$.

Forman, R. T. T., Land Mosaics: The Ecology of Landscapes and Regions, Cambridge, Cambridge University Press, 1995, 656 p.

Franklin, J. F., Forman, R. T. T, « Creating landscape patterns by forest cutting: ecological consequences and principles », Landscape Ecology, $n^{\circ}$ 1, 1987, p. 5-18.

Luginbühl, Y., « La demande sociale de paysage », rapport pour le Conseil national du paysage, 2001, 17 p., URL : https://www.ladocumentationfrancaise.fr/var/storage/rapports-publics/ 014000726.pdf

Luginbühl, Y., « Apollinien et Dionysiaque », dans Luginbühl, Y., (dir), Paysage méditerranéen, Milan, Electa, 1992, p. 24-31.

Paletto, A., Chincarini, M., Tosi, V., « Une analyse de la variabilité des volumes de bois mort observée dans les haies de Vénétie (Italie) », Revue forestière française, $\mathrm{n}^{\circ}$ 4, 2008, p. 437-450.

Riethmuller, T., Weiss, S., Chauvin, C., « Les chartes forestières de territoire : premier bilan », Ingénieries eau-agriculture-territoires, Antony, Irstea, 2003, p. 43-52.

Roche, P., Vennetier, M., Guyot, F., Moundy, P.-J., Bourguignon, V., « Dynamique périurbaine et composition floristique des petits îlots boisés en Provence calcaire ", Revue forestière française, $\mathrm{n}^{\circ}$ 5, 2008, p. 623-640.

Rougé, L., «Retour dans les espaces de la "captivité périurbaine" : diversification des parcours de vie et affirmation de logiques d'autonomisation », Sud-Ouest Européen, $\mathrm{n}^{\circ}$ 31, 2011, p. 43-53, mis en ligne en avril 2015, URL : http://journals.openedition.org/soe/828; DOI : https://doi.org/10.4000/ soe. 828

Schaepfer, R., « Un nouveau cadre pour l'aménagement des forêts », Revue forestière française, 2007, no 5, p. 515-523.

Sgard, A., Bonin, S., Davodeau, H., Dérioz, P., Paradis, S., et Toublanc, M., « Construire en commun par le paysage. Trois controverses paysagères relues à l'aune du bien commun ", Espaces et sociétés, $\mathrm{n}^{\circ}$ 175, 2018/4, p. 105-122, URL : https://www.cairn.info/revue-espaces-etsocietes-2018-4-page-105.htm

Sgard, A., «Le paysage dans l'action publique : du patrimoine au bien commun », Développement durable et territoires, vol. 1, no 2 , septembre 2010, URL : http://journals.openedition.org/ developpementdurable/8565; DOI : https://doi.org/10.4000/developpementdurable.8565

Veschambre, V., « Patrimoine : un objet révélateur des évolutions de la géographie et de sa place dans les sciences sociales ", Annales de géographie, n ${ }^{\circ}$ 656, 2007/4, p. 361-381, URL: https:// www.cairn.info/revue-annales-de-geographie-2007-4-page-361.htm 


\section{NOTES}

1. À savoir : CFT Luberon-Lure (synthèse des précédentes CFT Montagne de Lure et CFT Luberon), CFT Champsaur Valgaudemar, CFT des Maures, CFT du Pays A3V (Asses Verdon Vaïre Var), CFT Concors Sainte-Victoire, CFT Vésubie, CFT Ubaye-Pays de Seyne, CFT PNR de Camargue, CFT Pays dignois, CFT Pays d'Aix, CFT Artuby Verdon, CFT Baronnies provençales, CFT Grand Briançonnais des Écrins au Queyras, CFT Grand Estérel, CFT PNR Sainte-Baume.

2. http://documents.irevues.inist.fr/themes/4752/pdf/Presentation.pdf

3. Charte forestière de territoire du massif des Maures, 2010, disponible en ligne, URL : https:// drive.google.com/file/d/1muCF-c5E_cuqvK3LCXtXBuquOmIxk_Zu/view, consulté le 8 janvier 2020. Le titre du territoire varie selon les documents consultés, évoquant tantôt le massif, tantôt le Pays des Maures.

4. L'expression « débroussaillement en peau de léopard » fait référence à un débroussaillement de type alvéolaire, censé faciliter l'apparition d'une mosaïque de végétation et limiter l'impact visuel de cette opération.

5. Voir notamment la note argumentaire du Syndicat intercommunal pour la protection du massif de l'Esterel (SIPME) concernant le lancement de l'Opération grand Site Esterel de février 2018, disponible en ligne : https://www.charte-forestiere-esterel.com/images/PDF/noteargumentaire-OGS-Esterel_02.2018.pdf, consulté le 16 mai 2020.

\section{RÉSUMÉS}

L'article propose de réexaminer la place du paysage dans deux corpus de textes forestiers, entre 2006 et 2018, dans la continuité d'une thèse antérieure. Au travers des publications récentes de la Revue forestière française et des chartes forestières de territoire signées en région Provence-AlpesCôte d'Azur (Paca), la définition et l'application de la notion de paysage par les acteurs du monde forestier sont analysées. De plus en plus sollicitée d'un point de vue théorique comme outil d'analyse du territoire et de ses évolutions, la notion de paysage ne semble guère gagner en dimension opérationnelle. Les références au paysagisme d'aménagement, omniprésentes dans la décennie 1980-1990, se raréfient, tandis que l'écologie du paysage, mobilisée dans les travaux scientifiques sur la forêt, n'apparaît guère dans les documents plus opérationnels. En ce sens, les corpus étudiés apparaissent cohérents avec l'évolution plus large de la prise en compte du paysage dans les champs scientifiques et de l'aménagement des territoires. L'ensemble témoigne d'une évolution à bas bruit et sur le temps long, justifiant d'un point de vue méthodologique le « retour sur terrain » ou plus précisément sur « objet » à intervalles réguliers.

Following up on a thesis, this article re-examines the role of the landscape in two bodies of forestry documents dating from 2006 to 2018. Based on recent publications of the review Revue forestière française and forestry charters signed in the Provence-Alpes-Côte d'Azur (PACA) region, the definition and implementation of the notion of the landscape by forestry stakeholders is examined. This notion of the landscape is increasingly employed as a theoretical tool for analysing a territory and its evolution, however it does not seem to have acquired an operational dimension. References to landscape management, which were prevalent in the 1980s and 1990s, are becoming rarer, whereas landscape ecology, which is used in scientific research on forests, is hardly ever referred to in the more operational documents. In this sense, the documents studied 
appear to be consistent with the broader changes in the way the landscape is considered in scientific research and land use planning. The literature bears witness to gradual change over a long period of time which, from a methodological point of view, justifies going back into the "field" or more precisely re-examining the research "object" at regular intervals.

INDEX

Mots-clés : paysage, aménagement, paysagisme, territoire, géographie

Keywords : landscape, planning, landscaping, territory, geography

\section{AUTEUR}

\section{VÉRONIQUE FOURAULT-CAUËT}

Véronique Fourault-Cauët, géographe, maître de conférences, université Paris Nanterre, UMR Lavue 7218.

vfouraultcauet[at]parisnanterre[dot]fr 